\title{
Simplified Placental Localization
}

\author{
BRYAN M. HIBBARD,* M.D., PH.D., F.R.C.o.G.
}

\begin{abstract}
Ummary : Localization of the placenta by means of technetium-99m and a portable scintillation counter and ratemeter proved to be both simple and accurate. It could be performed by resident obstetric staff with minimal technical knowledge, and the radiation dosage was low.
\end{abstract}

\section{Introduction}

Modern obstetric practice has created a need for ancillary methods for accurate localization of the placental site. Placental localization by means of radioactive isotopes is now an established procedure in centres where medical radioisotope services are available. Such techniques, which are simple in principle, depend on the dispersion of a radioactive isotope, usually attached to a carrier molecule, through the maternal vascular system and on the detection of an area of increased radioactivity, resulting from increased vascularity, in the area of the placental bed.

The principal advantages of isotopic methods are: (i) the results are retiable in patients examined early in the third trimester and in association with such complications as malpresentation, polyhydramnios, and unsuspected pelvic tumours ; (2) the radiation hazard to the foetus can be reduced to less than $1 / 200$ of that incurred in taking two radiographs; and (3) the risks of a pelvic examination or of operative procedures associated with some radiological techniques, such as arteriography, are avoided.

Cost and clinical convenience are important considerations, and for these reasons attention has been given to the development of a technique employing a simple and inexpensive portable apparatus which can be used at the bedside by resident medical staff with minimal technical knowledge.

\section{Technetium-99m}

Many radioactive isotopes and carriers have been used for placental localization, and their relative properties discussed in detail elsewhere (Hibbard, 1966). In the present study technetium-99m was the isotope of choice because of its favourable characteristics, which may be summarized as follows: short physical half-life (six hours), monochromatic gamma-radiation in the optimal range $(140 \mathrm{KeV})$, lack of unnecessary betaradiation, radiochemical purity, ready availability from Mo-99 generator, and facility of labelling.

Molybdenum-99 generators are available commercially from the Radiochemical Centre, Amersham, and have an effective life of one to two weeks. Technetium- $99 \mathrm{~m}$ is obtained by elution, and the labelling of the albumin is carried out relatively simply by a modification of the method of Stern et al. (1965). The final product usually has a radioactive concentration of $400-450 \mu \mathrm{Ci} / \mathrm{ml}$. and a protein content of $1 \%$. The completeness of protein binding has been checked by paper chromatography and only rarely does the free pertechnetate exceed $0.2 \%$.

The metabolic behaviour and radiation dosimetry of technetium-99m-labelled albumin in pregnancy have been studied in detail (Herbert et al., 1969). There is satisfactory

* Senior Lecturer, Department of Obstetrics and Gynaecology, University of Liverpool, Liverpool L69 3BX. retention in the vascular system for the period necessary for scanning the uterus. Any free technetium liberated from the albumin is eliminated rapidly in the urine. The isotope can be detected in the bladder with an external counter about 20 minutes after intravenous injection. This may be a disadvantage in that it creates a potential source of error during scanning, especially if a protracted method such as autoscanning is used, but it does not cause any problems with the technique here described.

TABLE I.-Comparison of Radiation Dosages Received from Placental

\begin{tabular}{|c|c|c|c|}
\hline \multicolumn{4}{|c|}{ Localization Studies } \\
\hline & $\begin{array}{l}\text { Maternal } \\
\text { Gonads } \\
\text { (mrem) }\end{array}$ & $\begin{array}{l}\text { Foetal } \\
\text { Gonads } \\
\text { (mrem) }\end{array}$ & $\begin{array}{l}\text { Foetal } \\
\text { Thyroid } \\
\text { (mrem) }\end{array}$ \\
\hline 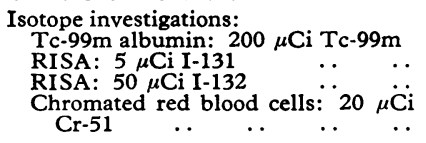 & $\begin{array}{l}7 \cdot 4 \\
15 \\
10 \\
12\end{array}$ & $\begin{array}{c}2 \cdot 4 \\
7 \\
5 \\
2-8\end{array}$ & $\begin{array}{c}3.6 \\
4,900 \\
130 \\
2-8\end{array}$ \\
\hline $\begin{array}{c}X \text {-radiography: Soft-tissue placento- } \\
\text { graphy (i exposures) or pelvic } \\
\text { arteriography (minimum) }\end{array}$ & 1,000 & 1,000 & 1,000 \\
\hline Natural background: Radiation per year & 100 & 100 & 100 \\
\hline
\end{tabular}

Exposure of the foetus to radiation is less with the amount of isotope normally used for this investigation than with any other radiological or radioisotope technique used at present for localizing the placenta. The foetal gonad dose is less than $1 \%$ of that resulting from a single $x$-ray film exposure and is the equivalent of only one to two weeks of natural background radiation. The suggestion that potassium perchlorate should be administered to block the maternal and foetal thyroid uptake of technetium has been proved unneccessary as a result of recent investigations of radiation dosimetry (Herbert et al., 1969).

\section{Apparatus}

A lightweight hand-held scintillation counter weighing $960 \mathrm{~g}$, similar to that specifically designed for in-vivo measurements with technetium- $99 \mathrm{~m}$, was used for the majority of the investigations (Herbert et al., 1965). It was fitted with a 3-mm. lead cylindrical collimator, so that the crystal face was recessed $5 \mathrm{~cm}$. from the face of the counter. Impulses were fed to an E.M.I. portable ratemeter, type RM2. This apparatus is battery-operated and transistorized throughout and weighs 2.5 $\mathrm{kg}$. The assembly was adjusted to give optimum sensitivity combined with stability, by means of conventional methods. A single control knob has "Off," " battery check," "E.H.T. check," and "sensitivity range" positions. An E.H.T. adjustment is also provided.

Recently a commercially produced assembly has become available and was used in the later investigations (Pitman Placental Localization Monitor Model 235) (Fig. 1). The general features are similar to the original apparatus, but certain refinements have been introduced. These include a time constant adjustment switch with two settings and a ratemeter calibration adjustment which allows count rates to be read direct as percentages of a selected standard, such as the precordial rate. This removes the need for subsequent conversion of readings. In addition there is an "isotope selector" switch 
with preset channel widths selected for optimal counting of technetium-99m, chromium-51, iodine-131, and Indium-113m.

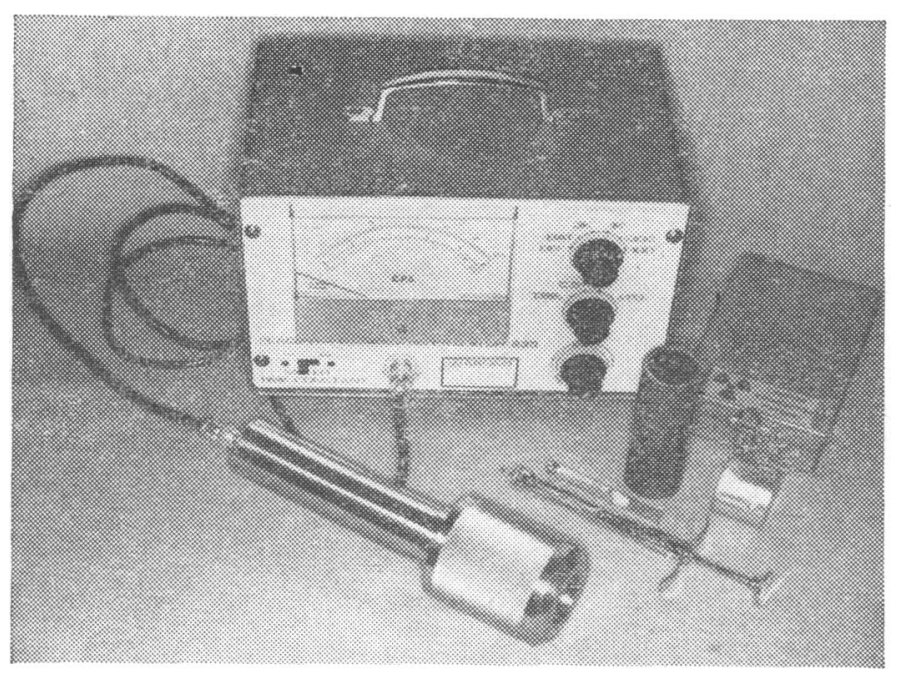

FIG. 1.-Equipment and materials for placental localization. The scintillation counter and ratemeter assembly (Pitman Model 235) is fully transistorized and battery-operated. The doses of technetium-99m transistorized and battery-operated. The doses of technetium-99m

\section{Technique}

The general technique is similar to that described previously in which radio-iodinated serum albumin (RISA) is used (Hibbard, 1961) and the investgation can be carried out at the patient's bedside (Fig. 2). $200 \mu \mathrm{Ci}$ of technetated albumin

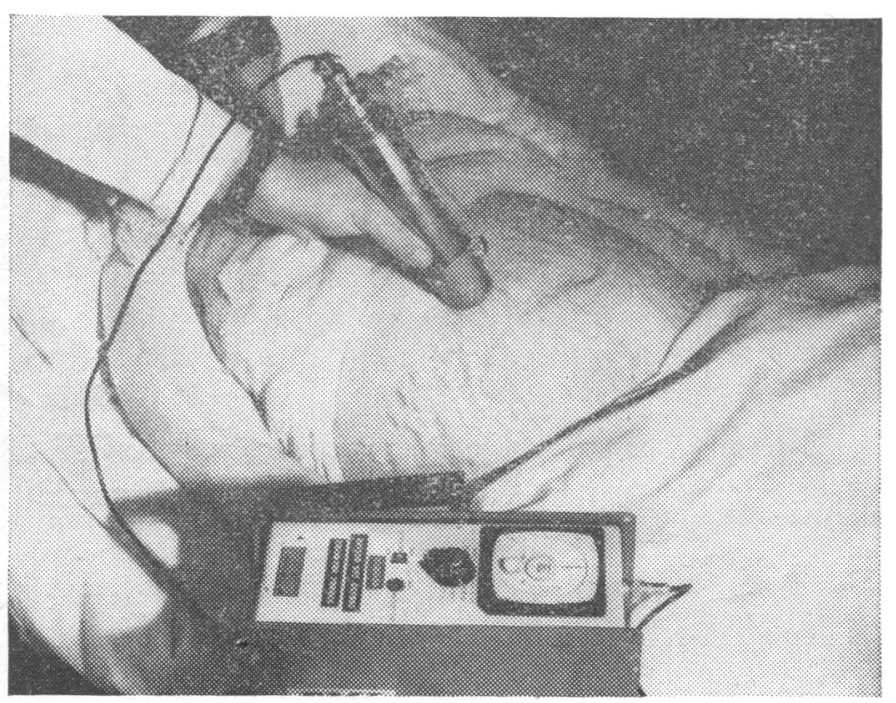

FIG. 2.-Placental localization performed at the bedside. The original assembly used in the majority of the cases described is shown (see text).

is administered intravenously in a volume of about $0.5 \mathrm{ml}$. Five to 10 minutes are allowed for even distribution in the vascular pool. With the apparatus described above count rates of the order of 1,000 c.p.s. - that is, full-scale deflection on the appropriate scale setting-are obtained over the sternum, which is use 1 as a reference point. The scale readings become reasonably stable within five seconds of positioning the counter. As background counts are low (2-3/second) no correction for this factor need be made. Also, as the investigation is completed within a few minutes no correction for radioactive decay is required.

The abdomen is then scanned, taking readings at points about $5 \mathrm{~cm}$. apart. The small size and manœuvrability of the counter head enable about three-quarters of the uterine wall to be scanned when the patient is lying supine. Manipulation of the patient is therefore unnecessary. Readings over the patient's back are of no value because of the amount of intervening tissue, and in practice are not required.

The results are expressed graphically, the readings being given as percentages of the sternal counts. With increasing experience, in most cases the placental site is very easily located so that detailed recordings of readings become unnecessary and the predicted site is merely illustrated diagrammatically on a form. This is accompanied by an assessment of the clinical prognosis based on the classification outlined previously (Hibbard, 1961)-namely:

Group 0. Placenta praevia definitely excluded.

Group 1. The placenta may just encroach on the lower uterine segment but the degree of placenta praevia is not sufficient to influence the clinical outcome.

Group 2. The placenta is definitely praevia but the degree cannot be exactly defined.

Group 3. A major degree of placenta praevia exists.

In addition, when the investigation is undertaken for other purposes appropriate relevant details of the placental location are given.

\section{Results}

Placental localization using the technique described was carried out on 324 patients at Mill Road Maternity Hospital, Liverpool, during the period July 1966 to December 1968. The majority of the investigations were performed by resident obstetric registrars as part of their normal clinical duties. The indications for investigation are shown in Table II.

TABLE II.-Indications for Placental Localization, Mill Road Maternity Hospital, Liverpool, fuly 1966 to December 1968

\begin{tabular}{|c|c|c|}
\hline Indication & Cases Investigated & $\begin{array}{l}\text { Placenta Praevia } \\
\text { of Clinically } \\
\text { Significunt Degree }\end{array}$ \\
\hline 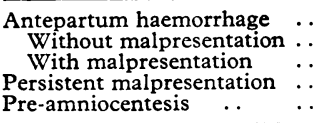 & $\begin{array}{rr}105 & 188 \\
83 & \\
& 113 \\
& 23\end{array}$ & $\begin{array}{c}35(18 \cdot 6 \%) \\
15(14 \cdot 3 \%) \\
20(24 \cdot 1 \%) \\
12(10 \cdot 6 \%) \\
1\end{array}$ \\
\hline & 324 & 48 \\
\hline
\end{tabular}

Eighteen per cent. of the examinations were carried out before 32 weeks' gestation and $39 \%$ before 36 weeks' gestation. In eight cases where examination was performed at 26 to 30 weeks the result was inconclusive and the test was repeated four weeks later.

Placenta praevia of a clinically significant degree was present in 47 of the cases investigated because of antepartum haemorrhage or malpresentation. In addition, a major degree of placenta praevia was an incidental finding in one patient who was examined before amniocentesis. Placenta praevia was found most commonly in those patients who were investigated because of haemorrhage associated with malpresentation of the foetus ( 20 out of 83 cases). Fifteen of the 105 patients who suffered haemorrhage but whose foetal presentation was normal were found to have placenta praevia. The condition occurred almost as commonly in the group of patients examined because of persistent malpresentation without haemorrhage (12 out of 113 cases). Overall, 13 of the 48 cases of placenta praevia $(27 \%)$ were diagnosed before haemorrhage occurred.

The results are considered primarily from the point of view of clinical management and outcome (Table III). Unless a clinical indication was present no attempt was made to locate the placental site by examination under anaesthesia before delivery of the infant or by exploration of the uterus after delivery. 
TARLE III.-Results of Placental Localization Studies in 301 Patients Investigated Because of Antepartum Haemorrhage and/or Persistent Malpresentation

\begin{tabular}{|c|c|c|c|}
\hline $\begin{array}{l}\text { Predicted } \\
\text { Group }\end{array}$ & Cases & $\begin{array}{l}\text { Errors of } \\
\text { Clinical } \\
\text { Importance }\end{array}$ & Comments \\
\hline 0 & $2: j$ & 1 & $\begin{array}{l}\text { Investigated at } 30 \text { weeks. } \\
\text { degree of placenta praevia } \\
\text { subsequently }\end{array}$ \\
\hline 1 & 27 & 1 & Examination under anaesthesia showed \\
\hline 2 & 31 & 5 & $\begin{array}{l}\text { major degree of placenta praevia } \\
\text { Examination under anaesthesia ex- } \\
\text { cluded significant degree of placenta } \\
\text { praevia. (In } 7 \text { further cases pos- } \\
\text { terior praevia was predicted but } \\
\text { subsequently excluded by lateral }\end{array}$ \\
\hline \multirow[t]{2}{*}{3} & 28 & 2 & $\begin{array}{l}\text { Spontaneous vaginal delivery with no } \\
\text { evidence of placenta praevia in either } \\
\text { case }\end{array}$ \\
\hline & 301 & *9 (3\%) & \\
\hline
\end{tabular}

* If the 7 cases in group 2 with initially erroneous predictions are included the incidence of significant errors is $16 / 301(5 \cdot 3 \%)$.

\section{Groups 0 and 1}

There were two errors of clinical importance concerning the 242 patients included in these categories. In one patient the examination was performed at 30 weeks' gestation, when there was no apparent evidence of placenta praevia, and in the other a minor degree of placenta praevia was diagnosed. In both of these patients the persistence of a malpresentation led to an examination under anaesthesia, when a major degree of placenta praevia which required caesarean section was found.

In 30 patients in these groups caesarean section was required for reasons other than placenta praevia, and in the remaining 210 cases vaginal delivery occurred. There was, however, no clinical evidence of placenta praevia in any of these cases.

\section{Group 2}

Five totally erroneous predictions in this group of 31 patients were made, placenta praevia being excluded by examination under anaesthesia. In addition, a further seven potential errors occurred, which could be ascribed to the isotope technique though the correct diagnosis was ultimately arrived at. In these cases a posterior placenta praevia was predicted, but because of uncertainty of the degree of praevia and the presence of a cephalic presentation standing lateral $x$-ray examination was carried out. This showed the head to be closely reiated to the sacral promontory, thereby excluding the presence of posterior placenta praevia of any significant degree. All of these patients had normal vaginal deliveries without further haemorrhage.

In the remaining 19 patients the presence of placenta praevia was confirmed either by examination under anaesthesia or at the time of caesarean section. In one case a low amniotomy was performed, there was severe bleeding late in labour, and the placenta was subsequently found to have two succenturiate lobes.

\section{Group 3}

A major degree of placenta praevia was suspected in 28 patients. The prediction was, however, incorrect in two cases. In both of these the foetal head engaged in the pelvis and spontaneous vaginal delivery occurred uneventfully. One patient went into labour prematurely at 31 weeks' gestation but had a rapid vaginal delivery. Pelvic examination immediately before delivery showed the edge of the placenta at the cervical os. The remaining 25 patients were all delivered by caesarean section without preliminary vaginal examination, and the placental site was confirmed in each case.
Overall, in the 301 patients investigated because of antepartum haemorrhage or parsistent malpresentation, or both, nine erroneous predictions $(3 \%)$ were made. If the seven cases in which posterior placenta praevia was originally predicted, but in which the diagnosis was subsequently excluded by lateral $x$-ray examination, are included, the overall failure rate becomes $5.3 \%$. A failure to diagnose placenta praevia occurred in 2 of the 48 patients who were subsequently shown to have a placenta praevia of a major degree-a failure rate of $4 \cdot 2 \%$.

\section{Pre-amniocentesis}

Twenty-three patients were examined as a preliminary to anminocentesis or intrauterine transfusion. In five cases the placenta was found to be at the proposed site for amniocentesis and therefore another area was selected. In no case was there any evidence that the amniocentesis needle was inserted through the placental bed. Maternal blood was examined by the Kleihauer technique after the operation in several cases, and no foetal cells were detected.

\section{Discussion}

Early confirmation or exclusion of placenta praevia by ancillary methods is important for medical, economic, and social reasons. A safe and reliable method for placental localization can be of real clinical value in assessing patients with antepartum haemorrhage, especially in those cases in which the initial haemorrhage is slight and conservative treatment is anticipated. In addition, many cases of placenta praevia can be diagnosed with confidence even before bleeding has occurred. Increasing use of amniocentesis as a diagnostic procedure and of intrauterine transfusion in the treatment of severe rhesus immunization also creates a need for placental localization.

With autoscanning equipment it is possible to obtain excellent delineation of the placental site, and such techniques are now quite widely used (MacAfee et al., 1964 ; Hibbard, 1966 ; Johnson et al., 1967 ; Thompson et al., 1967 ; Robertson et al., 1968 ; Walker and Kohorn, 1968). Sophisticated procedures of this nature, however, have several disadvantages from the clinical point of view. Because of the high cost and immobility of the apparatus and the need for skilled technical assistance the patient has to be transported to the isotope unit with the attendant risk-in cases of antepartum haemorrhage-of precipitating further bleeding. Furthermore, the investigation is relatively time-consuming, the usual scanning-time being 20 minutes. During this period the patient has to be immobile and in some methods described has to be in a prone position during the scan-a difficult and uncomfortable situation for a woman in advanced pregnancy.

The technique described in this paper fulfils most of the desired criteria for placental localization studies. Essentially it is simple, safe, rapid, and accurate. It can be performed at the bedside by resident obstetric staff and requires no exceptional degree of skill or technical knowledge.

The physical characteristics of technetium- $99 \mathrm{~m}$ are near optimal for in-vivo scanning procedures of this nature, and the good scanning properties are associated with low tissue radiation. It is thus possible to use a scintillation detector with a thin luminophor and with minimal collimation. This makes the apparatus light, portable, and inexpensive. A complete detecting apparatus for bedside use is obtained for less than $£ 250$.

The accuracy of the technique compares favourably with other methods of isotope placentography and with $x$-radiography. In a review of the reports on 2,256 placental localization studies in which radioactive isotopes (Hibbard, 1967) were used, errors of clinical significance were found in $60(2.7 \%)$ cases, with false-negative predictions in 19 out of $317(6 \%)$ cases of 
placenta praevia subsequently confirmed clinically. In a personal series of 672 patients there were $20(3 \%)$ errors of clinical significance. In the same review the results of $3,972 x$-ray examinations were analysed, errors of clinical significance being found in $221(5.6 \%)$. Failure to diagnose placenta praevia occurred in 30 out of 639 cases $(4 \cdot 7 \%)$.

When comparing these figures it must be remembered that in most of the isotope series very few cases were rejected as unsuitable for investigation, whereas restrictions were commonly imposed on the selection of patients in the $x$-ray series. It should also be recalled that, in the present series, as a matter of deliberate policy the majority of investigations were carried out by the resident obstetric staff, and the results reflect those which could be obtained in any obstetric unit that has the simple isotopic facilities required.

From a detailed study of the results it is evident that the principal source of error is in cases where the placenta is situated on the posterior uterine wall and its upper margin does not reach to the fundus of the uterus. In such cases there is a tendency, probably engendered by caution, to overestimate the degree of placenta praevia. This occurred particularly in patients examined early in the third trimester. In many cases the diagnosis was refuted by a repeat examination later in pregnancy, by a lateral $x$-ray cxamination of the pelvic brim if the foetal head presented, or solely by the clinical course of events. Though these errors never led to unnccessary major surgical procedures they did sometimes result in the patient being kept in hospital unnecessarily.

\section{REFERENCES}

Herbert, R. J. T., Kulke, W., and Shepherd, R. T. H. (1965). Postgraduate Medical fournal, 41, 656.

graduate Medical fournal, 41, 656.
Herbert, R. J. T., Hibbard, B. M., and Sheppard, M. A. (1969). foumal of Nuclear Medicine. In press.

Hibbard, B. M. (1961). Fournal of Obstetrics and Gynaccology of the British Commonwealth, 68, 481.

Hibbard, B. M. (1966). Clinical Obstetrics and Gynaccology, 9, 93.

Hibbard, B. M. (1967). Ph.D. thesis, University of Liverpool.

Johnson, P. M., Sciarra, J. J., and O'Leary, J. A. (1967). Radiology, 89, 321.

MacAfee, J. G., Stern, H. S., Fueger, G. F., Baggish, M. S., Holzman, G. B., and Zolle, I. (1964). Fournal of Nuclear Medicine, 5, 936 Robertson, E. G., Millar, D. G., and Day, M. J. (1968). fournal of Obstetrics and Gynaecology of the British Commonwealth, 75, 636. Stern, H. S. Zolle, I., and MacAfee, J. G. (1965). International fournal of Applied Radiation and Isotopes, 16, 283.

Thompson, W., Bell, T. K., and Pinkerton, J. H. M. (1967). British Medical fournal, 4, 390 .

Walker, R. H. S., and Kohorn, E. I. (1968). Fournal of Obstetrics and Gynaecology of the British Commonwealth, 75, 464.

\title{
Ampicillin Levels in Human Bile in the Presence of Biliary Tract Disease
}

\author{
P. R. MORTIMER,* M.B., B.S., M.C.PATH.; D. B. MACKIE, $\dagger$ M.CHIR., F.R.C.S. ; S. HAYNES, $\ddagger$ M.B., B.S., F.R.C.S.
}

British Medical fournal, 1969, 3, 88-89

\begin{abstract}
Cummary : Ampicillin levels were measured in the serum and in the bile from both the gall bladder and the common bile duct in patients undergoing surgery for biliary tract diseases. In patients with radiologically nonfunctioning gall bladders ampicillin was either not present or its concentration was lower than normal. Therapeutic levels were present in the common bile duct of all patients except those with obstruction of the common bile duct. Hence ampicillin fails appreciably to penetrate the obstructed viscus in obstructive biliary tract disease, and it is unlikely to be effective in treating infection associated with this.
\end{abstract}

\section{Introduction}

Ampicillin is frequently used in the treatment of acute cholecystitis and ascending cholangitis, as it is well recognized that in experimental animals the drug is actively concentrated in the liver, with resulting high levels in the bile. Stewart and Harrison (1961) showed that in rats the liver was capable of concentrating ampicillin to produce a level in the bile of up to 40 times the peak plasma concentration. Brown and Acred (1961), using dogs, found that the biliary concentration of ampicillin was up to 300 times that found in the blood. However, Ayliffe and Davies (1965), in the only previous study in man, found lower levels of ampicillin in gall bladder bile from patients with diseased biliary tracts than in their normal controls.

The object of the present study was to determine the concentration of ampicillin in the serum and in the bile from both the common bile duct and the gall bladder in patients with biliary tract disease one and a half to two hours after intramuscular injection.

\footnotetext{
* Lecturer.

+ Senior Registrar.

$¥$ Registrar.

Department of Bacteriology, Bland-Sutton Institute, Middlesex Hospital Medical School, and Department of Surgery, Middlesex Hospital, London W.1.
}

\section{Methods}

Twenty-three patients were studied. They were divided into three groups based on preoperative clinical, laboratory, and radiological findings.

Group 1.-Eleven patients with cholelithiasis and normal liver function tests, but without any evidence of obstruction of the cystic or common bile duct on oral or intravenous cholangiography.

Group 2.-Six patients with a blocked cystic duct, but no evidence of obstruction of the common bile duct or liver damage.

Group 3.-Six patients with obstructive jaundice.

No normal controls were included. It was considered unjustified to take samples of bile from patients who were not to have operations on the biliary tract.

All patients were given $500 \mathrm{mg}$. of ampicillin one and a half to two hours before the samples of bile and serum were obtained. At operation bile was obtained from the gall bladder through a fine needle, and where possible from the common bile duct through a fine catheter which was inserted via the cystic duct. A sample of systemic venous blood was obtained at the same time.

Ampicillin assays were performed by the diffusion method, described by Bennett et al. (1966), using Sarcina lutea as the test organism. The samples of bile and their controls were diluted in $\mathrm{M} / 20$ phosphate buffer $p H \quad 7 \cdot 0$. The samples of serum and their controls were diluted in pooled sterile normal human serum.

\section{Results}

Group 1.-The concentration of ampicillin found in the samples from patients of group 1, with cholelithiasis alone, are shown in Table I. The concentration of ampicillin in common bile duct samples was at least twice the serum level. The concentration in the gall bladder was in no case higher than in the common bile duct sample, and there was considerable variation in the level obtained. Some samples of gall bladder 